\title{
Comparative histological changes in the structure of the spleen and kidneys of experimental chickens exposed to the action of "Guidamis" as an adjuvant for vaccination against infectious bronchitis
}

\author{
Rezilya Asrutdinova ${ }^{1 *}$, Ildar Zalyalov ${ }^{1}$, Evgeny Kirillov ${ }^{1}$, Fannur Sunagatov ${ }^{2}$, and Alexander Dubovoy ${ }^{3}$ \\ ${ }^{1}$ Kazan State Academy of Veterinary Medicine named after N.E. Bauman, Kazan 420029, Russia \\ ${ }^{2}$ Main Veterinary Department of the Ministry of the Republic of Tatarstan, Kazan 420111, Russia \\ ${ }^{3}$ All-Russian Scientific Research Veterinary Institute of Poultry Farming of the Russian Agricultural Academy, St. Petersburg, \\ Lomonosov 198412, Russia
}

\begin{abstract}
The article provides a histological description of the internal organs - the spleen and kidneys of chickens exposed to the action of "Guidamis" as an adjuvant. A lysate was tested as part of a sample of the vaccine PB-07 against infectious bronchitis developed by researchers of the Department of Virology and Tumor Diseases of Birds of the Russian Agricultural Academy (Lomonosov). The experiments were carried out on Lohmann Brown chickens in the conditions of the Laishev branch of "Ak Bars" Poultry Company, the Republic of Tatarstan. The prolonged action of the vaccine against infectious bronchitis and the use of "Guidamis" as an adjuvant was manifested as an increase in the proliferation and differentiation of lymphoid tissues in the numerous lymph nodes. The use of the vaccine and the adjuvant had a weak reactogenic effect. In the spleen of control chickens, hyperplasia of lymphoid tissue cells is weakly expressed. In the periarterial zone of nodules of the thymus-dependent lymphocytes, the cell density is low. In the kidneys, for fourteen days after the vaccination against infectious bronchitis using Guidamis, the moderate granular dystrophy of the epithelium, mainly of individual distal tubules, and local moderate manifestations of edema of the capsule of the renal corpuscles were observed. In the kidneys, signs of pronounced edema of the capsule cavity, significant disturbances in the patency of the tubular network caused by swelling and partial desquamation of the epithelium, as well as the presence of epithelium and protein mass in the lumen of the tubules, were observed.
\end{abstract}

\section{Introduction}

The basis of the Russian poultry industry is large industrial enterprises, where the results of research are being implemented [1].

Economic indicators depend on the veterinary wellbeing of poultry farms. The level of the veterinarysanitary, ecological well-being of poultry enterprises is determined by the systemic interaction of all production units, especially veterinary and zootechnical departments [2-4].

The development of the poultry industry, new technologies for keeping and feeding poultry, as well as achievements of biotechnology increased the range of infectious diseases (infectious anemia of chickens, hepatitis E, astrovirus infection, etc.) caused by strains of new viruses (chicken infectious bronchitis - serovariants D 388, 4/91, QX, CR88, IT-02, etc.; Newcastle disease 7 serovariant; infectious bursal disease; metapneumovirus infection - serovariants $\mathrm{A}$ and $\mathrm{B} ; \mathrm{p}$ ovirus infection - Polish version; influenza - H5N1, H7N9), known viruses (pasteurellosis, hemophilosis), expansion of the species spectrum of pathogens (coccidiosis - E. necatrix, E. brunetti, E. mitis; salmonellosis - S. infantis, S.virchov ), reassortant viruses (chicken infectious bronchitis - isolate 062545/09 Swedish) [5-9]. The veterinary department of poultry farms should implement preventive measures, taking into account the technology of feeding young birds. Vaccines must be biologically harmless. None of the existing adjuvant vaccines is completely safe. The judgment on the safety of immunomodulators is based on toxicity studies conducted on laboratory and other annimals [10-14]. The most noticeable effect of adjuvants is manifested when they are used in combination with small doses of highly active antigens or with weak antigens. With simultaneous application of antigen, the effect of immunogenesis is more pronounced [15-18].

The focus of scientific research is the problem of selecting stimulating components to increase the antigenic activity of inactivated vaccines against viral diseases [19]. Therefore, the improvement and selection of stimulating components to increase the antigenic

*Corresponding author: roza509a@mail.ru 
activity of inactivated vaccines require the studies on new, more effective biological preparations.

\section{Materials and methods}

To determine the effectiveness of "Guidamis" as an adjuvant, 3 groups of chickens (20 chickens in each group) were formed. Group 1 was given a vaccine containing infectious bronchitis virus antigen (group 1 control), group 2 - a vaccine containing the chicken infectious bronchitis virus antigen and Guidamis lysate in the concentration of $1.0 \%$. Group 3 remained intact. Gidamis lysate was tested as part of a vaccine sample from strain PB-07 against chicken infectious bronchitis. The experiments were carried out on Lohmann Brown chickens. The adjuvant properties of the lysate were studied as part of a sample of the vaccine against infectious bronchitis of chickens, developed by researchers of the Department of Virology and Tumor Diseases of the Russian Agrarian Academy.

To perform a histomorphological analysis, pieces of the internal organs were fixed in a neutral aqueous $10 \%$ solution of formaldehyde and ethanol-formaldehyde (9: 1). Pieces of spleen, kidneys dehydrated in alcohols were compacted by pouring in paraffin. Stained histological sections 6-8 $\mu \mathrm{m}$ in thickness were stained with hematoxylin-eosin. When studying the microstructural changes in the internal organs and tissues, the most pronounced changes were photographed.

\section{Results}

On the 7th day after subcutaneous administration of a vaccine against infectious bronchitis using "Guidamis" as an adjuvant, in the moderately blood-filled red pulp with a pronounced structure of its reticular base, numerous small, oval, round-shaped lymph nodes were identified. In most of them, mainly in their central region, signs of transformation of reticular cells into dendritic forms with thickening of the processes, as well as the appearance of slightly differentiated and blast cells and single figures of mitosis were observed.

The periphery of the germinal centers of the lymph nodes was unevenly infiltrated by a layer of lymphoid cells differing in density and thickness, consisting mainly of large and medium forms. The boundaries of the mantle and marginal regions of the lymph nodes were poorly marked.

The periarterial region was distinguished by moderate edema, the reticular base and the presence of a small number of small lymphocytes. The central, trabecular arteries had marked profiles of the gaps, as well as the layers of their walls. In the vascular endothelium, bulging into the lumen of single pycnomorphic cells was observed.

On the 14th day after vaccination, a significant increase in the content of lymph nodes with the presence of germinal centers was observed. In most lymph nodes, post-vaccination immunomorphological rearrangement was manifested as a complicated structure of lymphoid formations. Around the central artery, the presence of cells with swollen processes of lymphocytes with hyperchromic nuclei and a narrow rim of the cytoplasm increased markedly. Small lymphocytes formed annular clusters of an uneven density and an irregular shape.

In the nodules, metaplasia and proliferation of lymphoid cells were enhanced. As a result of increased hyperplasia and transformation of lymphoid tissue cells in the nodules, clusters of lymphoblasts formed with a pronounced oxyphilic cytoplasm and a large pale nucleus, mitosis figures as a result of which the reticular base was hardly identified.

By this time, structural and functional zones and mantle regions developed in the lymph nodes. They were saturated with medium and small lymphocytes and macrophages. The external borders of the lymph nodes were distinguished both due to accumulations of small and medium lymphocytes, as well as squeezed reticular cells and fibers.

In the periarterial region, around the central artery, dense arrangements of numerous small lymphocytes were observed (Fig. 1).

In the walls of the trabecular, central arteries, the structure of the layers was preserved, and signs of edema of the perivascular regions were weakened.

Therefore, the prolonged exposure of the vaccine against infectious bronchitis of birds with the use of "Guidamis" as an adjuvant was manifested in the spleen as an increase in the proliferation and differentiation of lymphoid tissue in the numerous lymph nodes. The latter increased in volume.

On the 7th day after vaccination without using an adjuvant, numerous lymph nodes evenly located in the red pulp were identified in the spleen structure. They had a small volume, an indefinite shape with poorly pronounced functional zones. The germinal zones were clusters of dendritic cells and small blast cell forms, which are large forms with a poorly defined structure of nuclei. Only in individual lymph nodes, mitotic figures were identified. As a result of weak severity of cell proliferation and subsequent metaplasia, the germinal region of the nodules was separated from the reticular base of the red pulp, which was uneven in thickness and density.

Similar processes were observed in the periarterial region, only small lymphocytes were observed. In the walls of the trabecular, central arteries, signs of mucoid swelling were observed; in the surrounding tissue, edema and the reticular base were indicated.

On the 14th day of the experiment, after vaccination against infectious bronchitis without the use of an adjuvant, the content of lymph nodes with active proliferation and cell transformation increased significantly in the structure of the spleen.

As a result, germinal regions were observed. The cellular composition of the germinal centers of lymph nodes was significantly renewed due to an increase in the blast forms of cells and figures of mitosis, as well as large lymphocytes, but their density was lower than in the birds of the experimental group. 


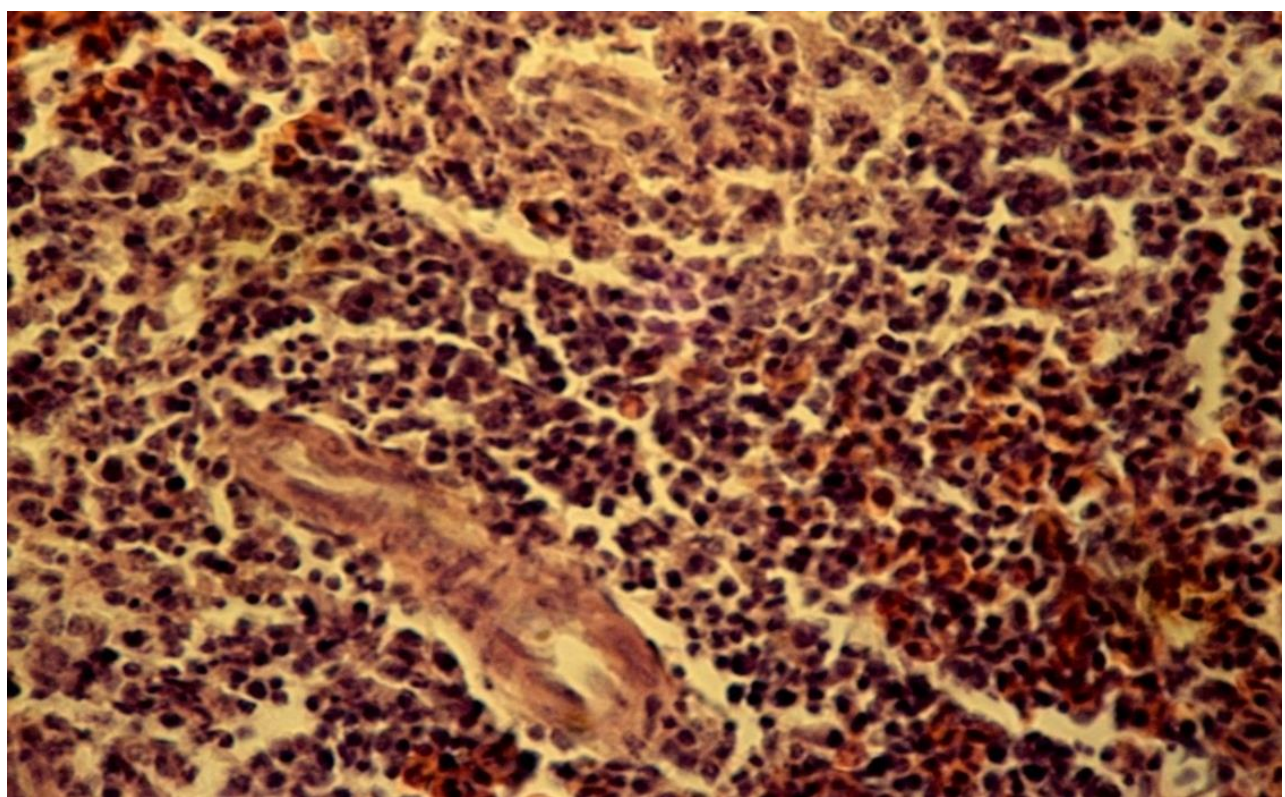

Fig. 1. The histology of the chicken spleen on the 14th day after vaccination using "Guidamis" as an adjuvant. Hematoxylin and eosin stain. Mg. 400

The severity of lymphoid tissue hyperplasia in the marginal and periarterial regions of the nodules was enhanced, but without clear borders of the marginal, mantle and periarterial zones due to the lower density of cell accumulations of small and medium lymphocytes, as well as macrophages.

In the structures of the organ stroma, the signs of mucoid swelling of the walls of the blood vessels of the trabeculae and lymph nodes and increased permeability of the walls of the capillaries were weakened.

Therefore, vaccination of birds against infectious bronchitis without the use of "Guidamis" is manifested as a lower level of severity of lymphoid tissue hyperplasia in the lymph nodes and their subsequent cellular transformation. A lower level of cell density was observed in the periarterial zone of the nodules of thymus-dependent lymphocytes (Fig. 2).

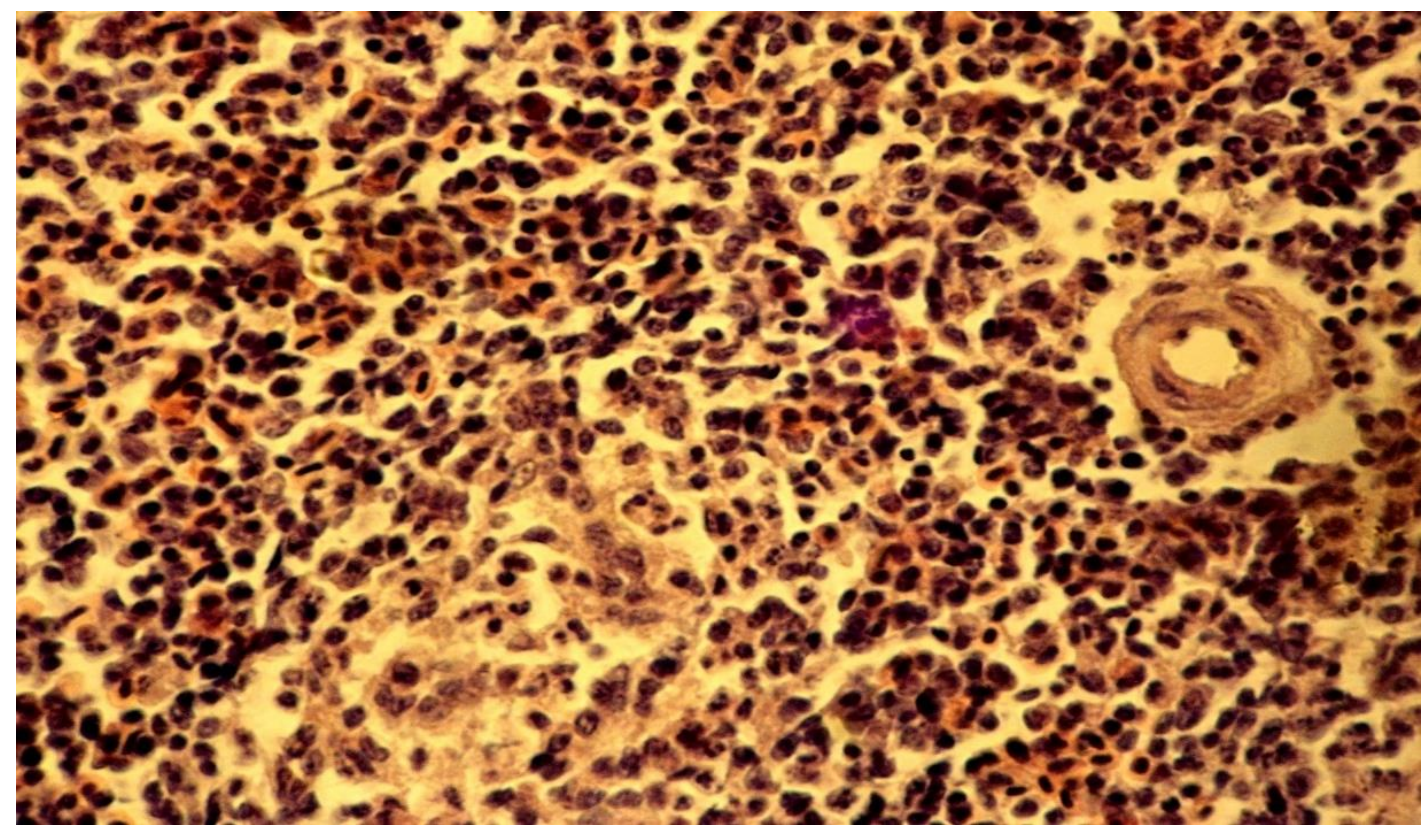

Fig. 2. The histology of the chicken spleen on the 14th day after vaccination without "Guidamis". Hematoxylin and eosin stain. Mg. 400.

In intact birds not exposed to Guidamis used along with the test vaccine against infectious bronchitis, signs of a delay in the formation of spleen lymph node structures were observed. They were small in number and located unevenly on the surface of the organ slice. The cellular composition of the lymph nodes was distinguished by the presence of reticulocytes with swollen processes and small cells. On the 7 th day, the shapes of mitosis were single, and on the 14th day their number increased slightly. A low level of proliferative activity of lymphoid tissue cells in the spleen was accompanied by a slowdown in the differentiation of the

Corresponding author: roza509a@mail.ru 
structure of lymph nodes. Structurally functional zones were poorly marked. These birds showed no signs of reactogenic action of the vaccine preparation in the structures of the stroma and parenchyma. Manifestations of local vascular disorders increased permeability of the vascular walls (Fig. 3).

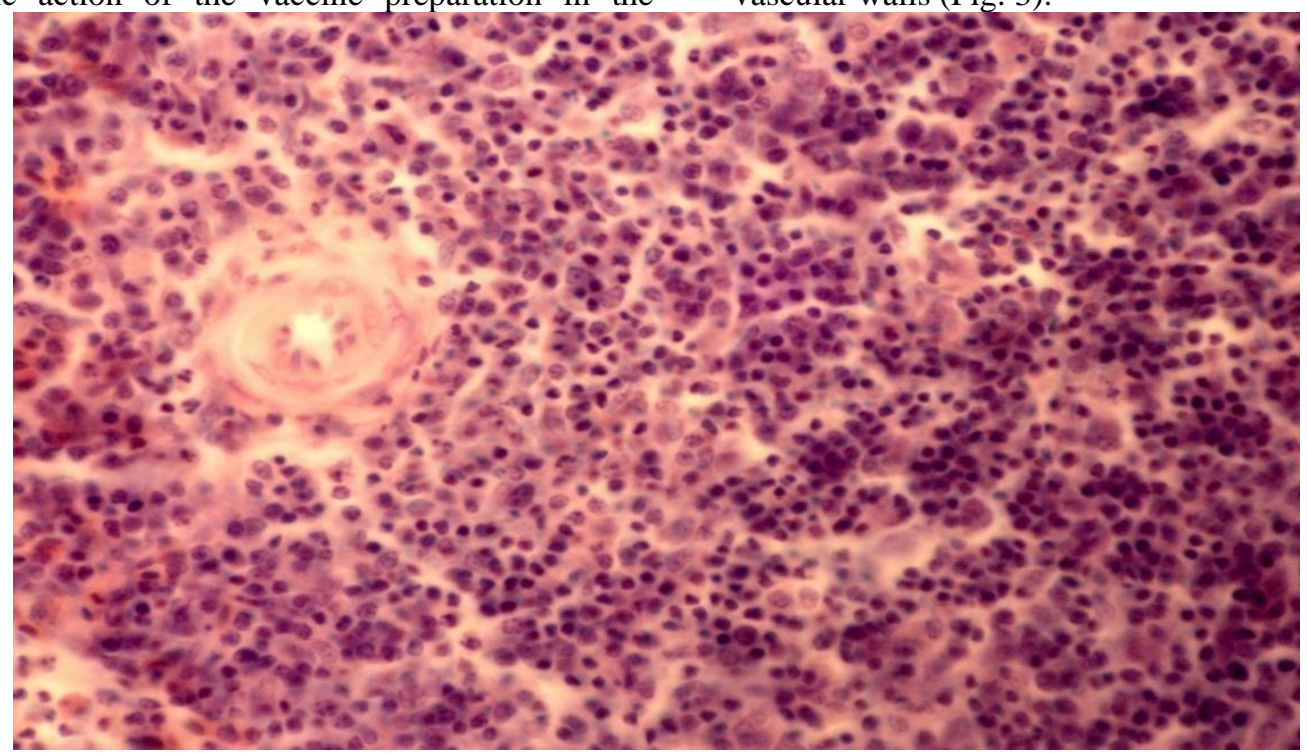

Fig. 3. The histology of the intact chicken kidney on the 14th day after vaccination. Hematoxylin and eosin stain. Mg. 400.

In the kidneys of experimental birds, on the fourteen day after vaccination (Fig. 4), moderate granular dystrophy of the epithelium, mainly of the individual distal tubules, and local moderate manifestations of edema of the capsule cavity of the renal corpuscles were observed. As a result, the level of blood supply to the organ parenchyma was not impaired. The throughput of the tubular organ network was preserved.

In the kidneys of birds, after vaccination without Guidamis, signs of pronounced edema of the capsule cavity, significant disturbances in the patency of the tubular network caused by swelling and partial desquamation of the epithelium, mainly the proximal region, as well as the presence of the epithelium and protein mass in the lumen of the tubules were observed (Fig. 5). Most of the capillaries in the vascular glomeruli and near the tubules did not have a pronounced lumen.

Consequently, in the kidneys of these birds, due to an increase in protein tubulonephrosis, there was a violation of the throughput of the tubular network and blood supply to the organ.

During the fourteen days of the experiment, the kidneys of intact birds (Fig. 6) showed signs of moderate granular dystrophy of the tubular epithelium, leading to local disturbances of glomerular filtration and patency of the tubular organ network.

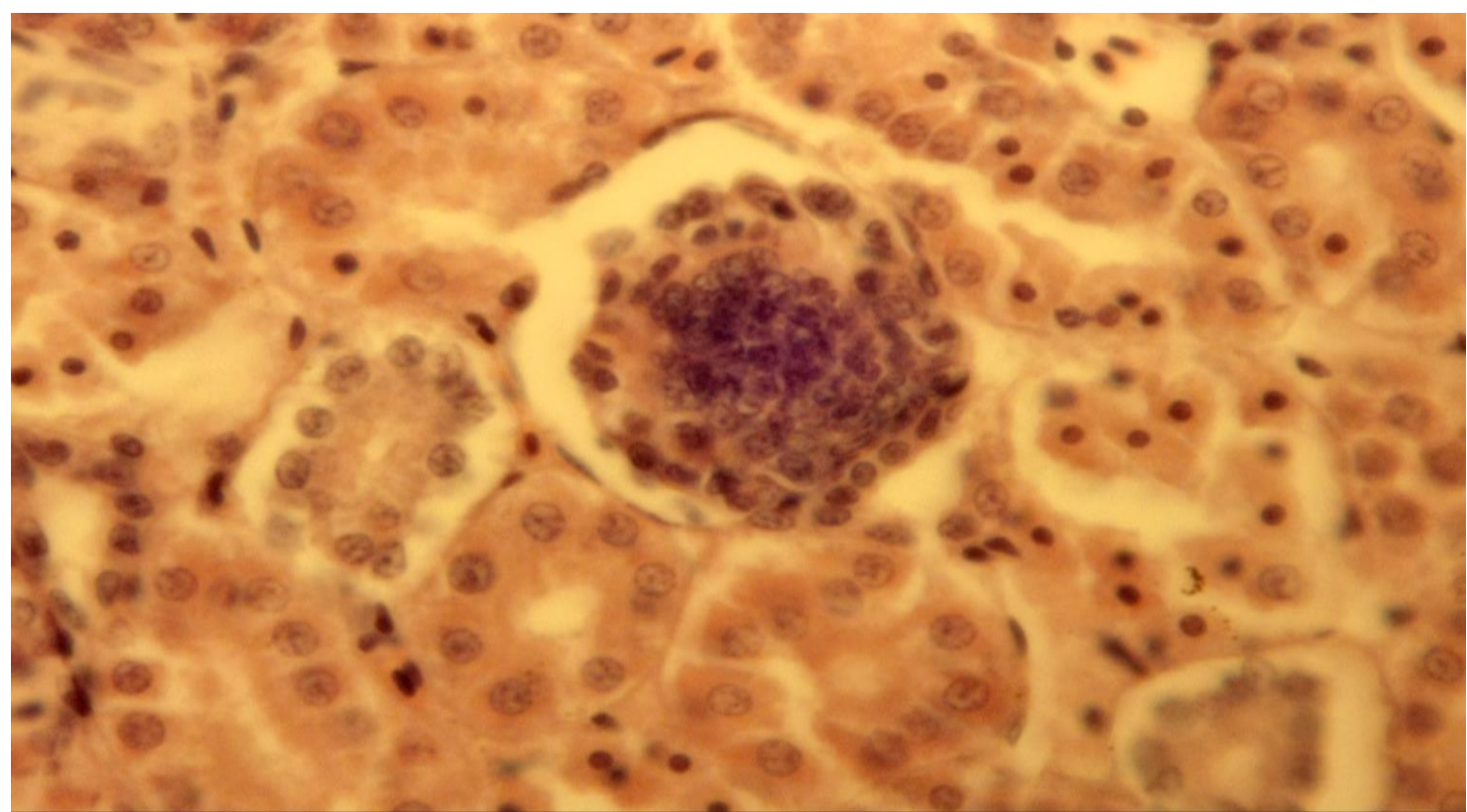

Fig. 4. The histology of the chicken kidney on the 14th day after vaccination using "Guidamis" as an adjuvant. Hematoxylin and eosin stain. Mg. 400. 


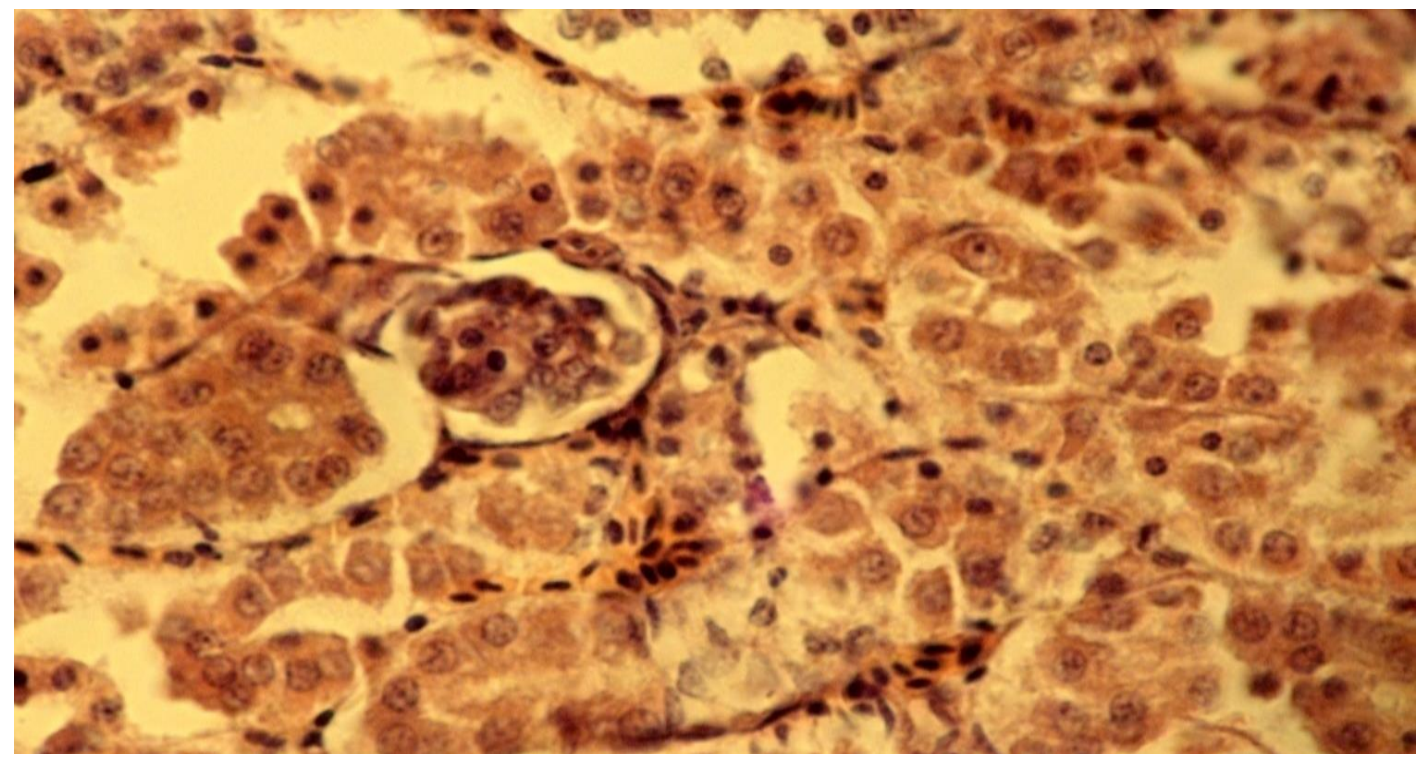

Fig. 5. The histology of the kidney of the chicken on the 14th day from the beginning of the experiment. Hematoxylin and eosin stain. Mg. 400.

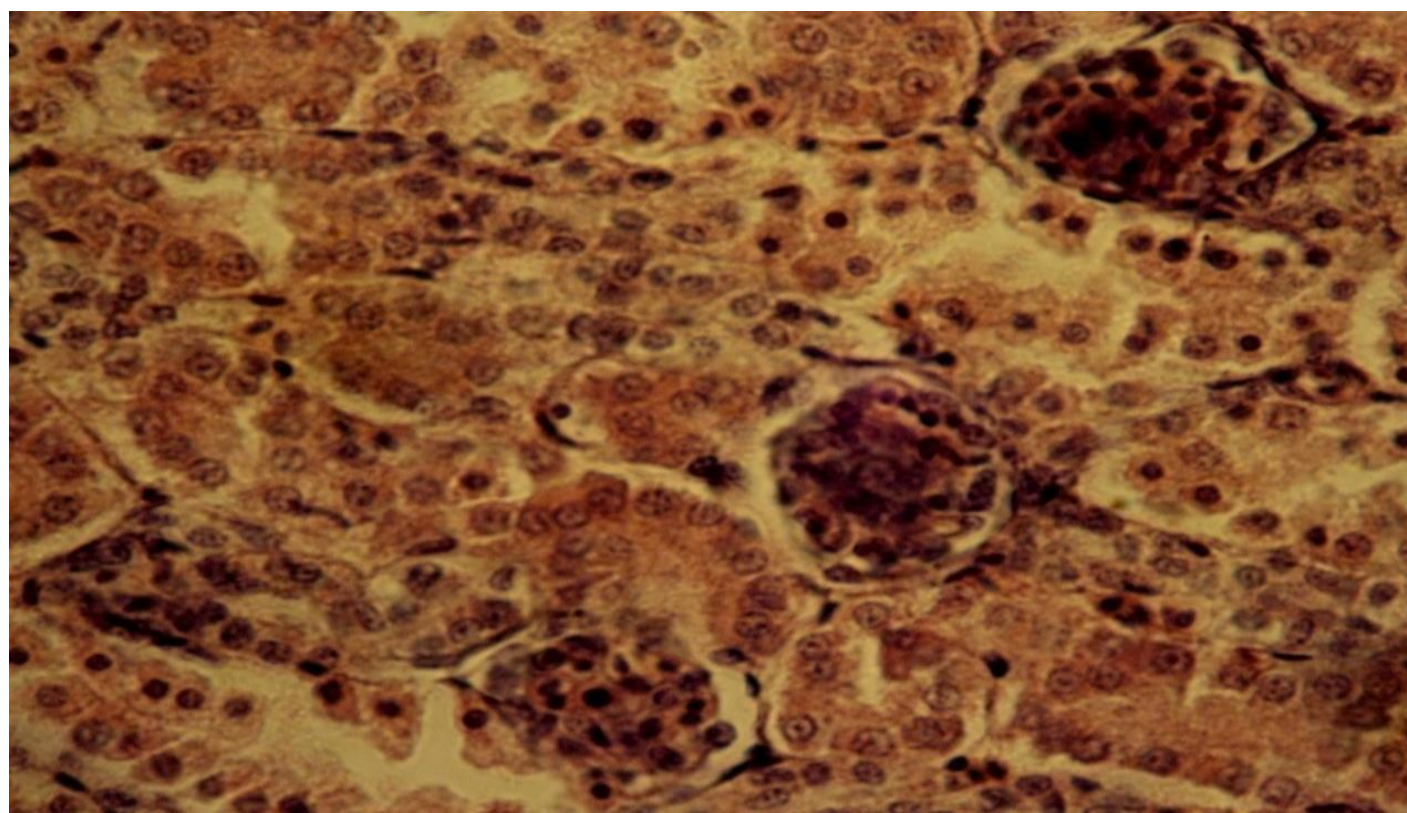

Fig. 6. The histology of the kidney of the intact chicken on the 14th day from the beginning of the experiment. Hematoxylin and eosin stain. Mg. 400.

\section{Conclusion}

Thus, histological studies of the spleen and kidneys of chickens indicate the stimulation of immunogenesis by Guidamis lysate used for vaccination against infectious bronchitis.

\section{References}

1. V. Fisinin, N. Zhuravel, A. Miftakhutdinov, Method for determining the effectiveness of new veterinary methods and tools in poultry farming, Veter. Med., 6, 14 (2018)

2. T. Lenkova, T. Egorova, I. Menshenin, Poultry, 4, 23 (2013)

3. N. Pridybaylo, Nanotechnology as a way to create new vaccines for poultry farming (Moscow, 2009)
4. F. Hoerr, Impact of subclinical immunosuppression on poultry production: clinical importance of immunosuppressive viruses, In: Proc. Symp. Impact of Subclinical Immunosuppression on Poultry Production (FL, Jacksonville, 2006)

5. V. Apatenko, M. Livoshchenko, The effect of viralbacterial infection on the immunocompetent system of birds (Kharkov, 1988)

6. N. Arkhipov, Veter. Med., 9, 26-28 (1983)

7. M. Dmitrieva, Farm Animals, 1, 77 (2014)

8. V. Apatenko, Associated bird infections (Kharkiv, 2002).

9. K. Ganapathy, V. Todd, P. Cargill et al., Avian Pathology, 6(35), 429-434 (2006) 
10. E. Ovseychik, Breeding broiler chickens using immunomodulators, Poultry farming, 41, 11-12 (2018)

11. I. Barinsky, A. Lazarenko, L. Alimbarova, The study of the effectiveness of using domestic immunomodulators, as well as their combined action with specific vaccines in experimental arbovirus infections, Immunol., 4, 181 (2012)

12. R. Asrutdinova, M.G. Sagitova, A.R. Kamaliev et al., Sci. notes of Kazan State Acad. of Veter. Med. named after N.E. Bauman, 217, 12-16 (2014)

13. S. Garipov, R. Asrutdinova, Sci. notes of Kazan State Acad. of Veter. Med. named after N.E. Bauman, 234, 73-78 (2018)

14. K. Zimin, The probiotic "Monosporin" is a stimulator of the humoral link in the immune response of animals and birds to bacterial infections, Poultry and poultry products, 2, 50 (2016)

15. F. Kamarudin, C. Gan, Molecular structure, chemical properties and biological activities of Pinto bean pod polysaccharide, Int. J. Biol. Macromol., 88, 280 (2016)

16. R. Ataullakhanov, R. Khaitov, Adjuvants in vaccines, Communication 1, Micro- and nanoparticles, Immunol., 32(1), 37 (2011)

17. S. Perekhodova, Yu. Patsula, Effect of thymalin on the formation of anti-brucellosis immunity in mice (Omsk, 1988)

18. A. Confer, R.J. Panciera, R.W. Fulton, M.J. Gentry, J.A. Rummage, Am. J. Vet. Res., 2(46), 342-347 (1985)

19. L. Kashkovskaya, N.N. Zhukova, M.I. Safarova et al., Veter., 4, 40-45 (2018) 